NASA Technical Memorandum 106644

ICOMP-94-15; CMOTT-94-8

\title{
A New Reynolds Stress Algebraic Equation Model
}

Tsan-Hsing Shih and Jiang Zhu

Institute for Computational Mechanics in Propulsion

and Center for Modeling of Turbulence and Transition

Lewis Research Center

Cleveland, Ohio

John L. Lumley

Cornell University

(NASA-TM-106644) A NEW REYNOLDS

N95-11953

Ithaca, New York

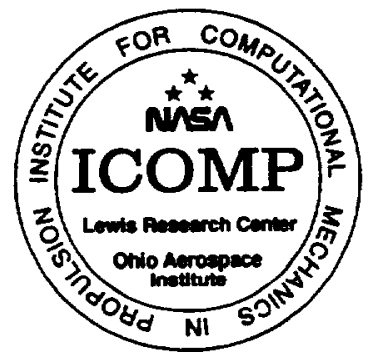




\title{
A New Reynolds Stress Algebraic Equation Model
}

\author{
Tsan-Hsing Shih and Jiang Zhu \\ Center for Modeling of Turbulence and Transition \\ Institute for Computational Mechanics in Propulsion \\ NASA Lewis Research Center, Cleveland, Ohio \\ John L. Lumley \\ Cornell University, Ithaca, New York
}

\begin{abstract}
A general turbulent constitutive relation (Shih and Lumley, 1993) is directly applied to propose a new Reynolds stress algebraic equation model. In the development of this model, the constraints based on rapid distortion theory and realizability (i.e. the positivity of the normal Reynolds stresses and the Schwarz' inequality between turbulent velocity correlations) are imposed. Model coefficients are calibrated using well-studied basic flows such as homogenous shear flow and the surface flow in the inertial sublayer. The performance of this model is then tested in complex turbulent flows including the separated flow over a backward-facing step and the flow in a confined jet. The calculation results are encouraging and point to the success of the present model in modeling turbulent flows with complex geometries.
\end{abstract}

\section{Introduction}

The present study concentrates on complex turbulent shear flows which are of great interest in propulsion systems. The particular flows presented in this paper are for the backward-facing step and the confined jet, both of which have complex structures. For example, a confined jet combines several types of flow structure and flow phenomena such as a shear layer, jet, recirculation, separation and reattachment. Accurate prediction of these flows is of great importance in all the key elements of engine design.

The turbulence model developed in this study is a Reynolds stress algebraic equation model which is based on a turbulent constitutive relation (Shih and Lumley, 1993), a result of rapid distortion theory (Reynolds, 1987) and the turbulent realizability principle (Schumann 1977, Lumley, 1978). The constitutive relation is obtained using the invariance theory in continuum mechanics. For flows including a passive scalar, this theory leads to a general constitutive relation for the Reynolds stress tensor $\overline{u_{i} u_{j}}$ in terms of the mean deformation rate tensor $U_{i, j}$ and the turbulent velocity and length scales characterized by the turbulent kinetic energy $k$ and its dissipation rate $\varepsilon$. Pope (1975) applied a similar constitutive relation to Rodi's algebraic Reynolds stress formulation (Rodi, 1972) in conjunction with the LRR second order closure model (Launder et al., 1975) and obtained an explicit algebraic 
expression for the Reynolds stresses for a two-dimensional mean flow field. Taulbee (1992, 1994) was able to extend this method to a general three-dimensional flow. Gatski and Speziale (1992) also applied this method in their algebraic Reynolds stress model. We note that in Rodi's algebraic Reynolds stress formulation, some assumptions, such as the constant anisotropy of Reynolds stresses and the neglect of turbulent transport of second moments, are in general not valid for most turbulent shear flows. These assumptions may bring large errors to turbulence modeling. In addition, the deficiency of existing second order closure models would also add extra errors to this type of model. In this study, Rodi's formulation is not considered. As an alternative, we directly impose the constraints based on rapid distortion (rotation) theory and realizability on the constitutive relation for the Reynolds stresses. As a result, a realizable algebraic expression for the Reynolds stresses in terms of the mean velocity gradient and the characteristic scales of turbulence is obtained for general three-dimensional turbulent flows. For turbulent scales, the standard $k-\varepsilon$ model transport equations are used in this study. Some model constants are calibrated using a well-studied homogeneous shear flow and a surface flow in the inertial sublayer and then tested in other complex flows. The model validation is made on the basis of applications to the rotational homogeneous shear flows simulated by Bardina et al. (1983), the two backward-facing step flows experimentally studied by Driver and Seegmiller (1985) and Kim et al. (1978) and the five cases of confined jets studied by Barchilon and Curtet (1964).

The calculations for complex flows are performed with a conservative finite volume method (Zhu, 1991b). Grid independent and low numerical diffusion solutions are obtained by using differencing schemes of second-order accuracy on sufficiently fine grids. For wallbounded flows, the standard wall function approach (Launder and Spalding, 1974) is used for wall boundary conditions. The results are compared in detail with the experimental data for both mean and turbulent quantities. The calculations using the standard $k-\varepsilon$ eddy viscosity model are also carried out for the purpose of comparison. The comparison shows that the present realizable Reynolds stress algebraic equation model significantly improves the predictive capability of $k-\varepsilon$ equation based models, expecially for flows involving massive separations or strong shear layers. In these situations, the standard eddy viscosity model overpredicts the eddy viscosity and, hence, fails to accurately predict shear stress, adverse pressure gradient, separation, reattachment, recirculation, etc. We find that the success of the present model in modeling complex flows is largely due to its effective eddy viscosity formulation which accounts for the effect of the mean deformation rate. According to the present model, the effective eddy viscosity will be significantly reduced by the mean deformation rate and maintained at a correct level to mimic the complex flow structures. 


\section{Turbulence model}

2.1 Constitutive relation. Constitutive relations for the Reynolds stresses were derived by several researchers (Pope, 1975, Yoshizawa, 1984 and Rubinstein and Barton, 1990). Shih and Lumley (1993) used the invariant theory in continuum mechanics and the generalized Cayley-Hamilton formulations (Rivlin, 1955) to derive a more (perhaps the most) general constitutive relation for the Reynolds stresses under the assumption that the Reynolds stresses are dependent only on the mean velocity gradients and the characteristic scales of turbulence characterized by the turbulent kinetic energy $k$ and its dissipation rate $\varepsilon$. This relation is

$$
\begin{aligned}
\overline{u_{i} u_{j}} & =\frac{2}{3} k \delta_{i j}+2 a_{2} \frac{K^{2}}{\varepsilon}\left(U_{i, j}+U_{j, i}-\frac{2}{3} U_{i, i} \delta_{i j}\right)+2 a_{4} \frac{K^{3}}{\varepsilon^{2}}\left(U_{i, j}^{2}+U_{j, i}^{2}-\frac{2}{3} \Pi_{1} \delta_{i j}\right) \\
& +2 a_{6} \frac{K^{3}}{\varepsilon^{2}}\left(U_{i, k} U_{j, k}-\frac{1}{3} \Pi_{2} \delta_{i j}\right)+2 a_{7} \frac{K^{3}}{\varepsilon^{2}}\left(U_{k, i} U_{k, j}-\frac{1}{3} \Pi_{2} \delta_{i j}\right) \\
& +2 a_{8} \frac{K^{4}}{\varepsilon^{3}}\left(U_{i, k} U_{j, k}^{2}+U_{i, k}^{2} U_{j, k}-\frac{2}{3} \Pi_{3} \delta_{i j}\right)+2 a_{10} \frac{K^{4}}{\varepsilon^{3}}\left(U_{k, i} U_{k, j}^{2}+U_{k, j} U_{k, i}^{2}-\frac{2}{3} \Pi_{3} \delta_{i j}\right) \\
& +2 a_{12} \frac{K^{5}}{\varepsilon^{4}}\left(U_{i, k}^{2} U_{j, k}^{2}-\frac{1}{3} \Pi_{4} \delta_{i j}\right)+2 a_{13} \frac{K^{5}}{\varepsilon^{4}}\left(U_{k, i}^{2} U_{k, j}^{2}-\frac{1}{3} \Pi_{4} \delta_{i j}\right) \\
& +2 a_{14} \frac{K^{5}}{\varepsilon^{4}}\left(U_{i, k} U_{l, k} U_{l, j}^{2}+U_{j, k} U_{l, k} U_{l, i}^{2}-\frac{2}{3} \Pi_{5} \delta_{i j}\right) \\
& +2 a_{16} \frac{K^{6}}{\varepsilon^{5}}\left(U_{i, k} U_{l, k}^{2} U_{l, j}^{2}+U_{j, k} U_{l, k}^{2} U_{l, i}^{2}-\frac{2}{3} \Pi_{6} \delta_{i j}\right) \\
& +2 a_{18} \frac{K^{7}}{\varepsilon^{6}}\left(U_{i, k} U_{l, k} U_{l, m}^{2} U_{j, m}^{2}+U_{j, k} U_{l, k} U_{l, m}^{2} U_{i, m}^{2}-\frac{2}{3} \Pi_{7} \delta_{i j}\right)
\end{aligned}
$$

where

$$
\begin{aligned}
& \Pi_{1}=U_{i, k} U_{k, i}, \quad \Pi_{2}=U_{i, k} U_{i, k}, \quad \Pi_{3}=U_{i, k} U_{i, k}^{2}, \\
& \Pi_{4}=U_{i, k}^{2} U_{i, k}^{2}, \quad \Pi_{5}=U_{i, k} U_{l, k} U_{l, i}^{2}, \quad \Pi_{6}=U_{i, k} U_{l, k}^{2} U_{l, i}^{2}, \\
& \Pi_{7}=U_{i, k} U_{l, k} U_{l, m}^{2} U_{i, m}^{2}
\end{aligned}
$$

Eq.(1) contains 11 undetermined coefficients which are, in general, scalar functions of various invariants of the tensors in question, for example, $S_{i j} S_{i j}$ (strain rate) and $\Omega_{i j} \Omega_{i j}$ (rotation rate) which are $\left(\Pi_{2}+\Pi_{1}\right) / 2$ and $\left(\Pi_{2}-\Pi_{1}\right) / 2$ respectively. The detailed forms of these scalar functions must be determined by other model constraints such as rapid distortion theory, realizability, and appropriate experimental data.

It is noticed that the standard $k-\varepsilon$ eddy viscosity model corresponds to the first two terms on the right hand side of Eq.(1). Both the two-scale DIA approach (Yoshizawa, 1984) and the RNG method (Rubinstein and Barton, 1990) also provided a similar relation which is the first five terms on the right hand side of Eq.(1). 
In this study, for the purpose of engineering application we truncate Eq.(1) to its quadratic tensorial form. The necessity of using higher order non-linear terms will be left for future study. To distinguish between the strain and rotation, we define

$$
\begin{aligned}
S_{i j}^{*} & =S_{i j}-\frac{1}{3} S_{k k} \delta_{i j}, & \Omega_{i j}^{*}=\Omega_{i j} \\
S_{i j}^{(2 *)} & =S_{i j}^{2}-\frac{1}{3} S_{k k}^{2} \delta_{i j}, & \Omega_{i j}^{(2 *)}=\Omega_{i j}^{2}-\frac{1}{3} \Omega_{k k}^{2} \delta_{i j}
\end{aligned}
$$

where

$$
\begin{aligned}
& S_{i j}=\frac{1}{2}\left(U_{i, j}+U_{j, i}\right), \quad \Omega_{i j}=\frac{1}{2}\left(U_{i, j}-U_{j, i}\right) \\
& S_{i j}^{2}=S_{i k} S_{k j} \quad \Omega_{i j}^{2}=\Omega_{i k} \Omega_{k j}
\end{aligned}
$$

From the above definitions, we have the following relations:

$$
S_{i i}^{*}=0, \quad S_{i i}^{(2 *)}=0, \quad \Omega_{i i}^{(2 *)}=0
$$

For later use, we further define

$$
\begin{aligned}
S^{*} & =\sqrt{S_{i j}^{*} S_{i j}^{*}}, \quad \Omega^{*}=\sqrt{\Omega_{i j}^{*} \Omega_{i j}^{*}}, \quad S^{(2 *)}=\sqrt{S_{i j}^{(2 *)} S_{i j}^{(2 *)}} \\
W^{*} & =\frac{S_{i j}^{*} S_{j k}^{*} S_{k i}^{*}}{\left(S^{*}\right)^{3}} \quad W^{(2 *)}=\frac{S_{i j}^{(2 *)} S_{j k}^{(2 *)} S_{k i}^{(2 *)}}{\left(S^{(2 *)}\right)^{3}} \\
U^{*} & =\sqrt{S_{i j}^{*} S_{i j}^{*}+\Omega_{i j}^{*} \Omega_{i j}^{*}}, \quad U^{(2 *)}=\sqrt{S_{i j}^{(2 *)} S_{i j}^{(2 *)}+\Omega_{i j}^{(2 *)} \Omega_{i j}^{(2 *)}}
\end{aligned}
$$

Using Eqs.(3.1-3.4), the truncated equation (1) can be written as

$$
\begin{aligned}
\overline{u_{i} u_{j}} & =\frac{2}{3} k \delta_{i j}-C_{\mu} \frac{k^{2}}{\varepsilon} 2 S_{i j}^{*}+C_{1} \frac{k^{3}}{\varepsilon^{2}} 2\left(S_{i j}^{(2 *)}+\Omega_{i j}^{(2 *)}\right) \\
& +C_{2} \frac{k^{3}}{\varepsilon^{2}}\left(S_{i j}^{(2 *)}-\Omega_{i j}^{(2 *)}-S_{i k}^{*} \Omega_{k j}^{*}+\Omega_{i k}^{*} S_{k j}^{*}\right) \\
& +C_{3} \frac{k^{3}}{\varepsilon^{2}}\left(S_{i j}^{(2 *)}-\Omega_{i j}^{(2 *)}+S_{i k}^{*} \Omega_{k j}^{*}-\Omega_{i k}^{*} S_{k j}^{*}\right)
\end{aligned}
$$

2.2 Rapid distortion constraint. Reynolds (1987) and Mansour et al. (1991) studied the effect of rapid rotation on turbulence using rapid distortion theory (RDT). It was shown that there is no effect of the rapid mean rotation on the isotropic turbulence. This result provides a constraint for Eq.(4). For rotating flows with $S_{i j}=0$, Eq.(4) becomes

$$
\begin{aligned}
b_{i j} & =\frac{\overline{u_{i} u_{j}}}{2 k}-\frac{1}{3} \delta_{i j} \\
& =\frac{k^{2}}{2 \varepsilon^{2}} \Omega_{i j}^{(2 *)}\left(2 C_{1}-C_{2}-C_{3}\right)
\end{aligned}
$$


From the result of RDT for the isotropic turbulence, $b_{i j}$ should remain zero under rapid mean rotation and, therefore, we must require $2 C_{1}=C_{2}+C_{3}$. As a result, Eq.(4) becomes

$$
\begin{aligned}
\overline{u_{i} u_{j}} & =\frac{2}{3} k \delta_{i j}-C_{\mu} \frac{k^{2}}{\varepsilon} 2 S_{i j}^{*} \\
& +C_{2} \frac{k^{3}}{\varepsilon^{2}}\left(2 S_{i j}^{(2 *)}-S_{i k}^{*} \Omega_{k j}^{*}+\Omega_{i k}^{*} S_{k j}^{*}\right) \\
& +C_{3} \frac{k^{3}}{\varepsilon^{2}}\left(2 S_{i j}^{(2 *)}+S_{i k}^{*} \Omega_{k j}^{*}-\Omega_{i k}^{*} S_{k j}^{*}\right)
\end{aligned}
$$

2.3 Realizability. Realizability (Schumann,1977, Lumley,1978), defined as the requirement of the non-negativity of turbulent normal stresses and Schwarz' inequality between any fluctuating quantities, is a basic physical and mathematical principle that the solution of any turbulence model equation should obey. It also represents the minimal requirement to prevent a turbulence model from producing unphysical results. In the following, this principle will be applied to the relation of Eq.(5) to obtain constraints on its coefficients $C_{\mu}, C_{1}$ and $C_{2}$. The same procedure together with the RDT constraint can be also applied to the full equation (1).

Turbulence models often produce unphysical results under some extreme situations. For example, under a rapid mean strain the turbulent energy component in the strain direction will be rapidly reduced and a non-realizable model often drives that energy component to a negative value and under a high mean shear the turbulent shear stress will rapidly increase and a non-realizable model often overpredicts this increase such that the Schwarz' inequality will be violated. The commonly used $k-\varepsilon$ eddy viscosity model with a constant $C_{\mu}=0.09$ :

$$
\overline{u_{i} u_{j}}=\frac{2}{3} k \delta_{i j}-C_{\mu} \frac{K^{2}}{\varepsilon} 2 S_{i j}^{*}
$$

is one such unrealizable model. In this model, the energy component $\overline{u_{1}^{2}}$ will become negative when $S_{11}^{*} k / \varepsilon>1 / 0.27$ and the correlation coefficient between $u_{1}$ and $u_{2}$ will exceed unity when $S_{12}^{*} k / \varepsilon>1 / 0.27$ for a pure mean shear flow (which has only one non-zero component $S_{12}^{*}$ ).

To make eddy viscosity model Eq.(6) realizable, the coefficient $C_{\mu}$ cannot be a constant. It must vary with the mean flow deformation rate. To determine its appropriate formulation, we may use the following realizability constraints:

$$
\begin{aligned}
& \overline{u_{\alpha}^{2}} \geq 0 \quad(\alpha=1,2,3) \\
& \frac{{\overline{u_{\alpha} u_{\beta}}}^{2}}{\overline{\overline{u_{\alpha}^{2}}} \overline{u_{\beta}^{2}}} \leq 1 \quad(\alpha=1,2,3 ; \beta=1,2,3)
\end{aligned}
$$


Reynolds (1987) used the constraint of Eq.(7.1) to formulate the coefficient $C_{\mu}$ which ensures positive normal stresses. Shih et al. (1993) also imposed Eq.(7.1) on their Reynolds stress algebraic equation model. Here, we will follow the method of Reynolds and use both Eqs.(7.1) and (7.2) to determine the coefficients in Eq.(5).

In the principal axes of $S_{i j}^{*}$ (note, $S_{i i}^{*}=0$ ), we may write:

$$
S_{i j}^{*}=\left(\begin{array}{ccc}
1 & 0 & 0 \\
0 & -\frac{1+a}{2} & 0 \\
0 & 0 & -\frac{1-a}{2}
\end{array}\right) S_{11}^{*}
$$

The invariant $\mathrm{S}^{*}$ and $W^{*}$ defined in Eq.(3.4) can be calculated as

$$
S^{*}=\left|S_{11}^{*}\right| \sqrt{\frac{3+a^{2}}{2}}, \quad W^{*}=\frac{\frac{3}{4}\left(1-a^{2}\right)}{\left(\frac{3+a^{2}}{2}\right)^{3 / 2}}
$$

In addition, noting that in the principal axes of $S_{i j}^{*}$ the off-diagonal terms of $S_{i j}^{(2 *)}$ are also zero and that $S_{i i}^{(2 *)}=0$, we may write

$$
S_{i j}^{(2 *)}=\left(\begin{array}{ccc}
1 & 0 & 0 \\
0 & -\frac{1+b}{2} & 0 \\
0 & 0 & -\frac{1-b}{2}
\end{array}\right) S_{11}^{(2 *)}
$$

The invariants $S^{(2 *)}$ and $W^{(2 *)}$ are

$$
S^{(2 *)}=\left|S_{11}^{(2 *)}\right| \sqrt{\frac{3+b^{2}}{2}}, \quad W^{(2 *)}=\frac{\frac{3}{4}\left(1-b^{2}\right)}{\left(\frac{3+b^{2}}{2}\right)^{3 / 2}}
$$

According to Eq.(5), the energy component $\overline{u_{1}^{2}}$ in the principal axes of $S_{i j}^{*}$ is

$$
\overline{u_{1}^{2}}=\frac{2}{3} k-C_{\mu} \frac{k^{2}}{\varepsilon} 2 S_{11}^{*}+\left(C_{2}+C_{3}\right) \frac{k^{3}}{\varepsilon^{2}} 2 S_{11}^{(2 *)}
$$

Now let us consider the contraction case in which $S_{11}^{*}>0, S_{11}^{(2 *)}>0$ and $\overline{u_{1}^{2}}$ will decrease due to the contraction strain. Using Eqs.(8) and (9), we obtain

$$
\overline{u_{1}^{2}}=\frac{2}{3} k-C_{\mu} \frac{k^{2}}{\varepsilon} 2 S^{*} \sqrt{\frac{2}{3+a^{2}}}+\left(C_{2}+C_{3}\right) \frac{k^{3}}{\varepsilon^{2}} 2 S^{(2 *)} \sqrt{\frac{2}{3+b^{2}}}
$$

Now, applying the constraint Eq.(7.1) and allowing the component $\overline{u_{1}^{2}} \rightarrow 0$ but remains positive as $S^{*} \rightarrow \infty$ and $S^{(2 *)} \rightarrow \infty$. To satisfy this constraint, we may let

$$
\begin{aligned}
C_{\mu} & =\frac{A}{A_{0}+\sqrt{\frac{18}{3+a^{2}}} U^{* \frac{k}{\varepsilon}}} \\
C_{2}+C_{3} & =\frac{B}{A_{1}+\sqrt{\frac{18}{3+b^{2}}} U^{(2 *)}\left(\frac{k}{\varepsilon}\right)^{2}}
\end{aligned}
$$


and

$$
A-B=1
$$

Following Reynolds (1987), let

$$
A_{s}^{*}=\sqrt{\frac{18}{3+a^{2}}}, \quad A_{s}^{(2 *)}=\sqrt{\frac{18}{3+b^{2}}}
$$

Using Eqs.(8) and (9), $A_{s}^{*}$ and $A_{a}^{(2 *)}$ can be determined by the following equations:

$$
\begin{aligned}
\left(A_{s}^{*}\right)^{3}-\frac{9}{2} A_{s}^{*}-9 W^{*} & =0 \\
\left(A_{s}^{(2 *)}\right)^{3}-\frac{9}{2} A_{s}^{(2 *)}-9 W^{(2 *)} & =0
\end{aligned}
$$

It can be shown that the positive root of the above equations can be obtained when the values of $W^{*}$ and $W^{(2 *)}$ are between $-1 / \sqrt{6}$ and $1 / \sqrt{6}$ which correspond to axisymmetric expansion and axisymmetric contraction respectively. The appropriate roots are

$$
\begin{aligned}
A_{s}^{*} & =\sqrt{6} \cos \phi, & \phi & =\frac{1}{3} \arccos \left(\sqrt{6} W^{*}\right) \\
A_{s}^{(2 *)} & =\sqrt{6} \cos \phi, & \phi & =\frac{1}{3} \arccos \left(\sqrt{6} W^{(2 *)}\right)
\end{aligned}
$$

Eqs.(11.3) and (11.4) show that the values of $A_{s}^{*}$ and $A_{s}^{(2 *)}$ are between $\sqrt{6} / 2$ and $\sqrt{6}$. The model coefficients can be now written as

$$
\begin{aligned}
C_{\mu} & =\frac{A}{A_{0}+A_{s}^{*} U^{* \frac{k}{\varepsilon}}} \\
C_{2}+C_{3} & =\frac{B}{A_{1}+A_{s}^{(2 *)} U^{(2 *)}\left(\frac{k}{\varepsilon}\right)^{2}}
\end{aligned}
$$

The further determination of $A, B, A_{0}$ and $A_{1}$ should be carried out by using the constraint of Eq.(7.2) and the experimental data from well-studied turbulent flows such as homogeneous shear flows and channel flows.

Here, we try to propose a simple as possible but workable model (which contains the property of anisotropy) for engineering application and leave the more complete model form of Eqs.(5), (12) and (13) for future study. To do that, we choose $A=1$, then $B$ must be equal to zero and $C_{3}=-C_{2}$. As a result, Eq.(5) becomes

$$
\overline{u_{i} u_{j}}=\frac{2}{3} k \delta_{i j}-C_{\mu} \frac{k^{2}}{\varepsilon} 2 S_{i j}^{*}+2 C_{2} \frac{k^{3}}{\varepsilon^{2}}\left(-S_{i k}^{*} \Omega_{k j}^{*}+\Omega_{i k}^{*} S_{k j}^{*}\right)
$$


It is obvious that this model satisfies the constraint Eq.(7.1). To apply the constraint Eq.(7.2), we use a pure shear flow with only one non-zero component $U_{1,2}$ (i.e., $S_{12}^{*}=\Omega_{12}^{*}>$ 0 ) which can be considered as the most extreme case for satisfying Schwarz' inequality. For this flow, the relevant Reynolds stresses are

$$
\begin{aligned}
\overline{u_{1} u_{2}} & =-C_{\mu} \frac{k^{2}}{\varepsilon} 2 S_{12}^{*} \\
\overline{u_{1}^{2}} & =\frac{2}{3} k+4 C_{2} \frac{k^{3}}{\varepsilon^{2}} S_{12}^{*} \Omega_{12}^{*} \\
\overline{u_{2}^{2}} & =\frac{2}{3} k-4 C_{2} \frac{k^{3}}{\varepsilon^{2}} S_{12}^{*} \Omega_{12}^{*}
\end{aligned}
$$

Now using the constraint of Eq.(7.2), we may find a formulation for $C_{2}$ :

$$
C_{2}=\frac{\sqrt{1-9 C_{\mu}^{2}\left(\frac{S^{*} k}{\varepsilon}\right)^{2}}}{C_{0}+6 \frac{S^{*} k}{\varepsilon} \frac{\Omega^{*} k}{\varepsilon}}
$$

where

$$
C_{\mu}=\frac{1}{A_{0}+A_{s}^{*} \frac{U^{* k}}{\varepsilon}}
$$

The model represented by Eqs.(14), (16.1) and (16.2) is quite simple but has several advantages compared to the standard $k-\varepsilon$ eddy viscosity model of Eq.(6). First, the present model is fully realizable. It will not produce negative energy components and will not violate the Schwarz' inequality between turbulent velocities. Second, the effective eddy viscosity, defined as $\overline{u_{\alpha} u_{\beta}} / 2 S_{\alpha \beta}^{*}$, is anisotropic as it should be. Finally, the present model contains the effect of mean rotation on Reynolds stresses with a proper behavior that matches the RDT result: the mean rotation will not affect the isotropic turbulence.

There are still two model constants, $A_{0}$ and $C_{0}$, that need to be determined. We may use Eqs.(15) for the homogeneous shear flow or the surface flow in the inertial sublayer. According to these flows, $A_{0}$ and $C_{0}$ are chosen as

$$
A_{0}=6.5, \quad C_{0}=1.0
$$

With the values of $A_{0}$ and $C_{0}$ in Eq.(17), the model of Eq.(14) gives $b_{12}=-0.156, b_{11}=$ $-b_{22}=0.123$ for Tavoularis and Corrsin's (1981) homogeneous shear flow at $U_{1,2} k / \varepsilon=6.08$ and gives $b_{12}=-0.122, b_{11}=-b_{22}=0.14$ for the direct numerical simulation of channel flow (Kim, 1990) in the inertial sublayer at $U_{1,2} k / \varepsilon=3.3$. These results show that the present model gives reasonable anisotropy of Reynolds stresses for both the homogeneous shear flow and the boundary layer flow compared to the standard $k-\varepsilon$ eddy viscosity model which gives $b_{11}=b_{22}=0$ for both the flows and gives $b_{12}=-0.273$ for the homogeneous shear flow and 
$b_{12}=-0.149$ for the boundary layer flow. Detailed comparisons with the experimental and DNS data are shown in Table 1 for the homogeneous shear flow of Tavoularis and Corrsin (1981) and in Table 2 and Figure 1 for the channel flow of Kim (1990).

Table 1. Anisotropy in the homogeneous shear flow

\begin{tabular}{|c|c|c|c|}
\hline & experiment & standard & present \\
\hline$b_{12}$ & -0.142 & -0.273 & -0.156 \\
\hline$b_{11}$ & 0.202 & 0. & 0.123 \\
\hline$b_{22}$ & -0.145 & 0. & -0.123 \\
\hline
\end{tabular}

Table 2. Anisotropy in the channel flow

\begin{tabular}{|c|c|c|c|}
\hline & DNS data & standard & present \\
\hline$b_{12}$ & -0.145 & -0.149 & -0.122 \\
\hline$b_{11}$ & 0.175 & 0. & 0.14 \\
\hline$b_{22}$ & -0.145 & 0. & -0.14 \\
\hline
\end{tabular}

2.4 Model equations. Here we summarize the equations and the models which will be used for applications in the next section. For incompressible flows, the mean flows are governed by the following equations

$$
\begin{gathered}
U_{i, i}=0 \\
U_{i, t}+\left(U_{j} U_{i}-\nu U_{i, j}+\overline{u_{i} u_{j}}\right)_{, j}=-\frac{p, i}{\rho}
\end{gathered}
$$

where the Reynolds stresses will be modeled by Eq.(14):

$$
\overline{u_{i} u_{j}}=\frac{2}{3} k \delta_{i j}-C_{\mu} \frac{k^{2}}{\varepsilon} 2 S_{i j}^{*}+2 C_{2} \frac{k^{3}}{\varepsilon^{2}}\left(-S_{i k}^{*} \Omega_{k j}^{*}+\Omega_{i k}^{*} S_{k j}^{*}\right)
$$

and $C_{\mu}, C_{2}$ are determined by Eq.(16):

$$
C_{\mu}=\frac{1}{A_{0}+A_{\varepsilon}^{*} \frac{U^{* k}}{\varepsilon}}, \quad C_{2}=\frac{\sqrt{1-9 C_{\mu}^{2}\left(\frac{S^{*} k}{\varepsilon}\right)^{2}}}{C_{0}+6 \frac{S^{*} k}{\varepsilon} \frac{\Omega^{*} k}{e}}
$$

where

$$
A_{0}=6.5, \quad C_{0}=1.0
$$

Two quantities in Eq.(14), the turbulent kinetic energy $k$ and its dissipation rate $\varepsilon$, remain to be determined. At the present time, we use the standard $k-\varepsilon$ model equations which are

$$
\begin{aligned}
& k_{, t}+U_{j} k_{, j}=\left[\left(\nu+\frac{\nu_{t}}{\sigma_{k}}\right) k_{, j}\right]_{, j}-\overline{u_{i} u_{j}} U_{i, j}-\varepsilon \\
& \varepsilon_{, t}+U_{j} \varepsilon_{, j}=\left[\left(\nu+\frac{\nu_{t}}{\sigma_{\varepsilon}}\right) \varepsilon_{, j}\right]_{, j}-C_{\varepsilon 1} \frac{\varepsilon}{k} \overline{u_{i} u_{j}} U_{i, j}-C_{\varepsilon 2} \frac{\varepsilon^{2}}{k}
\end{aligned}
$$


where

$$
\nu_{t}=C_{\mu} \frac{k^{2}}{\varepsilon}
$$

The coefficients $C_{\varepsilon 1}, C_{\varepsilon 2}, \sigma_{k}$ and $\sigma_{\varepsilon}$ assume their standard values:

$$
C_{\varepsilon 1}=1.44, \quad C_{e 2}=1.92, \quad \sigma_{k}=1, \quad \sigma_{e}=1.3
$$

\section{Applications}

3.1 Rotating homogeneous shear flow. The present model is able to mimic the effect of the mean rotation rate on the turbulence. A test case is the rotating homogeneous shear flow which was studied by Bardina et al. (1983) using the large eddy simulation (LES) method. The effect of solid body rotation or the rotation of the reference frame on the turbulence must be appropriately incorporated in Eq.(14) through the terms containing $\Omega_{i j}^{*}$. In addition, the coefficients $C_{\mu}$ and $C_{2}$ should be also modified by the rotation rate of the reference frame, $\omega_{i}$ (angular velocity). Particularly, the $U^{*}$ in Eq.(16.2) is modified by

$$
U^{*}=\sqrt{S_{i j}^{*} S_{i j}^{*}+\bar{\Omega}_{i j}^{*} \tilde{\Omega}_{i j}^{*}}
$$

where

$$
\begin{aligned}
& \tilde{\Omega}_{i j}^{*}=\Omega_{i j}^{*}-2 \varepsilon_{i j k} \omega_{k} \\
& \Omega_{i j}^{*}=\bar{\Omega}_{i j}-\varepsilon_{i j k} \omega_{k}
\end{aligned}
$$

where $\bar{\Omega}_{i j}$ is the mean rotation rate viewed in the rotating reference frame. Figure 2 is the configuration of the flow being tested where $\Omega=\omega_{3}$ and $\bar{\Omega}_{12}=S_{12}^{*}=\frac{1}{2} \partial U / \partial y=$ $S / 2$. Figures $3(\mathrm{a})-3(\mathrm{c})$ show the evolution of the turbulent kinetic energy $k / k_{0}$ with the nondimensional time, $S t$, at the rotation rates of $\Omega / S=0,0.5$ and -0.5 , respectively, where $k_{0}$ is the initial turbulent kinetic energy, $S$ is the mean strain rate and $\Omega$ is the angular velocity of the reference frame. The calculations were performed with a fourth order Runge-Kutta scheme. The initial condition corresponding to the isotropic turbulence used in LES with $\varepsilon_{0} / S k_{0}=0.296$ was adopted for all the three cases. The results from both the present model and the standard $k-\varepsilon$ model (hereafter referred to as SKE) are compared with LES results in figures $3(\mathrm{a})-3(\mathrm{c})$. These figures show the ability of the present model to simulate the effect of the large rotation rate on turbulence. Note that the SKE model gives the same results as for the no rotation case because it cannot account for the effect of rotation on the evolution of turbulence. 


\subsection{Backward-facing step flows.}

Numerical procedure. For computational convenience, the non-dimensional form of the governing equations is solved, in which

$$
\begin{aligned}
& <x_{i}>=\frac{x_{i}}{L_{\text {ref }}}, \quad\left\langle U_{i}>=\frac{U_{i}}{U_{\text {ref }}}, \quad\left\langle p>=\frac{p}{\rho U_{\text {ref }}^{2}},\right.\right. \\
& <k>=\frac{k}{U_{\text {ref }}^{2}}, \quad<\varepsilon>=\frac{\varepsilon L_{\text {ref }}}{U_{\text {ref }}^{3}}, \quad\left\langle\nu_{t}>=\frac{\nu_{t}}{U_{\text {ref }} L_{\text {ref }}}\right.
\end{aligned}
$$

where $<>$ refers to a non-dimensional quantity, and $L_{\text {ref }}$, and $U_{\text {ref }}$ are the reference length and velocity, respectively. Accordingly, the flow Reynolds number is defined by

$$
R e=\frac{L_{r e f} U_{r e f}}{\nu}
$$

Hereafter, all the quantities will be of the non-dimensional form so that $<>$ will be dropped for simplicity.

In the steady-state and two dimensional cases $\left(x_{1}=x, x_{2}=y\right)$, the transport equations (19), (20) and (21) can be written in the following general form

$$
\left[U \phi-\left(\frac{1}{R e}+\frac{\nu_{t}}{\sigma_{\phi}}\right) \phi_{, x}\right]_{, x}+\left[V \phi-\left(\frac{1}{R e}+\frac{\nu_{t}}{\sigma_{\phi}}\right) \phi_{, y}\right]_{, y}=S_{\phi}
$$

where $\phi$ stands for the dependent variables: $U, V, k$ and $\varepsilon . S_{\phi}$ is the source term for each corresponding equation.

The numerical method used to solve the system of equations (27) is a finite-volume procedure. It uses a non-staggered grid with all the dependent variables being stored at the geometric center of each control volume (Figure 4). The momentum interpolation procedure of Rhie and Chow (1983) is used to avoid spurious oscillations usually associated with the non-staggered grid, and the pressure-velocity coupling is handled with the SIMPLEC algorithm (Van Doormal and Raithby, 1984). To ensure both accuracy and stability of the numerical solution, the convection terms are approximated by a second-order accurate and bounded differencing scheme (Zhu, 1991a), and all the other terms by the conventional central differencing scheme. As a result, the discretized counterpart of equation (27) can be cast into the following linearized form

$$
\phi_{C} \sum_{l} A_{l}=\sum_{l} A_{l} \phi_{l}+S_{C}
$$

where the coefficients $A_{l}(l=W, E, S, N)$, which relate the principal unknown $\phi_{C}$ to its neighbours $\phi_{l}$ (Figure 4), result from the discretization of the left-hand side terms of equation (27). The convection scheme used ensures that $A_{l} \geq 0$ so that the resulting coefficient matrix 
is always diagonally dominant. The strongly implicit procedure of Stone (1968) is used to solve the system of algebraic equations. The iterative solution process is considered to be converged when the maximum normalized residue of all the dependent variables is less than $10^{-4}$. The details of the present numerical procedure are given in Rodi et al. (1989) and Zhu (1991b).

Numerical results. Application is made to the two backward-facing step flows experimentally studied by Kim, Kline and Johnston (1978) and Driver and Seegmiller (1985), from here on referred to as KKJ- and DS-cases, respectively. Figure 5 shows the flow configuration and the Cartesian coordinate system. Table 3 gives the flow parameters for both cases; here the experimental reference free-stream velocity $U_{\text {ref }}$ and step height $H_{3}$ are taken as the reference quantities for non-dimensionalization.

Table 3. Flow parameters

\begin{tabular}{|c|c|c|c|c|c|c|c|}
\hline Case & $\operatorname{Re}$ & $\delta$ & $L_{s}$ & $L_{e}$ & $H_{s}$ & $H_{d}$ & $U_{\text {ref }}$ \\
\hline DS & 37423 & 1.5 & 10 & 40 & 1 & 8 & 1 \\
\hline KKJ & 44737 & 0.6 & 10 & 40 & 1 & 2 & 1 \\
\hline
\end{tabular}

Three types of boundaries are present, i.e. inlet, outlet and solid wall. At the inlet, the experimental data are available for the streamwise mean velocity $U$ and the turbulent normal stresses $\overline{u u}$ and $\overline{v v} . \mathrm{k}$ is calculated from these $\overline{u u}$ and $\overline{v v}$ with the assumption that

$$
\overline{w w}=\frac{1}{2}(\overline{u u}+\overline{v v})
$$

and $\varepsilon$ by

$$
\varepsilon=\frac{C_{\mu}^{3 / 4} k^{3 / 2}}{L}, \quad L=\min (0.41 \Delta y, 0.085 \delta)
$$

where $\Delta y$ is the distance from the wall and $\delta$ is the boundary-layer thickness given in Table 3. At the outlet, the streamwise derivatives of the flow variables are set to zero. Influences of both inlet and outlet conditions on the solution are examined by changing the locations $L$, and $L_{e}$, and it has been found that in both cases, the distances given in Table 3 are already sufficiently far away from the region of interest. In the earlier stage of this work, we tested several low Reynolds number $k-\varepsilon$ models including those of Chien (1982), Lam and Bremhorst (1981), Launder and Sharma (1974), Shih and Lumley (1992), and Yang and Shih (1992), but none of them was found to be able to yield satisfactory solutions for the skin friction along the bottom wall. Similar findings were also reported in Avva et al. (1990), Shuen (1992) and So and Lai (1988). Therefore in this work, we use the standard wall function approach (Launder and Spalding, 1974) to bridge the viscous sublayer near the wall.

Two sets of non-uniform computational grids are used to examine the grid dependence of the solution; they contain $110 \times 52$ (coarse) and $199 \times 91$ (fine) points for the KKJ-case and 
$106 \times 56$ (coarse) and $201 \times 109$ (fine) points for the DS-case. Figures $6(\mathrm{a})$ and $6(\mathrm{~b})$ show the friction coefficient $C_{f}$ at the bottom wall calculated with the SKE model and the present model; also included in figure 6(a) are the experimental data for the DS-case, but no such data are available for the KKJ-case. It can be seen that the grid refinement does produce some differences for the results of the present model, more noticeable in the KKJ-case, and this is also the case for the SKE results. This indicates that the solutions obtained on the coarse grids are not sufficiently close to the grid-independent stage. Recently, Thangam and Hur (1991) have conducted a highly-resolved calculation for the KKJ-case. They have found that quadrupling a $166 \times 73$ grid leads to only a minimal improvement. Therefore, the present results on the fine grids can be considered as grid-independent. For the DScase, the fine grid computations with the SKE model and present model required 703 and 691 iterations, and took approximately 7.1 and 9 minutes of CPU time on the Cray YMP computer. In the following, only the fine grid results are presented.

The wall friction coefficient $C_{f}$ is a parameter that is very sensitive to the near-wall turbulence modeling. It is $C_{f}$ that the various low Reynolds number $k-\varepsilon$ models tested predict much worse than those using wall functions. However, the influence of the near-wall turbulence modeling is mainly restricted to the near-wall regions. It is seen from figure 6(a) that both the SKE model and the present model largely underpredict the negative peak of $C_{f}$, pointing to limited accuracy of the wall function approach in the recirculation region.

The computed and measured reattachment points are compared in Table 4. They are determined in the calculation from the point where $C_{f}$ goes to zero. The reattachment point is a critical parameter which has often been used to assess the overall performance of turbulence models as well as numerical procedures. Table 4 clearly demonstrates the significant improvement obtained with the present model. It is important to mention that this improvement is mainly due to the behavior of $C_{\mu}$ in the present model, and that the anisotropic behavior of the turbulent stresses only makes a marginal contribution to it.

Table 4. Comparison of the reattachment points

\begin{tabular}{|c|c|c|c|}
\hline Case & measurement & SKE & PRESENT \\
\hline DS & 6.1 & 4.99 & 5.80 \\
\hline KKJ & $7 \pm 0.5$ & 6.35 & 7.27 \\
\hline
\end{tabular}

Figures $7(\mathrm{a})$ and $7(\mathrm{~b})$ show the comparison of computed and measured static pressure coefficients $C_{p}$ along the bottom wall. In both cases, the SKE model is seen to predict premature pressure rises which is consistent with its underprediction of the reattachment lengths.

The streamwise mean velocity $U$ profiles are shown in figures $8(\mathrm{a})$ and $8(\mathrm{~b})$ at four different cross-sections. Here, the differences between the results of the SKE model and present model are not substantial, as compared to other flow variables. However, the present model shows somewhat slower recovery in the vicinity of the reattachment point. We notice 
that such a slow recovery also exists in the Reynolds stress model prediction by Obi et al. (1989). Further downstream, say at $x=20$ in figure $8(a)$, the results of the two models nearly coincide with each other.

Finally, the comparisons of predicted and measured turbulent stresses $\overline{u^{2}}, \overline{v^{2}}$ and $\overline{u v}$ are shown in figures 9 and 10 at various $x$-locations. In the KKJ-case, no experimental data for the turbulent stresses are available in the recirculation region, and the reattachment point was found in the experiment to move forward and backward continuously around seven step heights downstream of the step, leaving an uncertainty of \pm 0.5 step height for the reattachment length. This also points to some uncertainty in the measured turbulent quantities in the recovery region. On the other hand, the experimental data in the DS-case should be considered more reliable because of the smaller uncertainty of the reattachment location, indicating a smaller unsteadiness of the flow. The SKE model gives unrealistic results about normal Reynolds stresses: $\overline{v^{2}}>\overline{u^{2}}$ at all the locations. In contrast, the present model gives at least qualitatively correct results due to the non-linear terms in Eq.(14) which increase $\overline{u^{2}}$ while decreasing $\overline{v^{2}}$, leading to an overall improvement in both $\overline{u^{2}}$ and $\overline{v^{2}}$ results.

3.3 Confined Jets. The general features of confined jets measured by Barchilon and Curtet (1964) are sketched in figure 11. At the entrance, two uniform flows, a jet of larger velocity and an ambient stream of smaller velocity, are discharged into a cylindrical duct of diameter $D_{0}$. The inlet flow conditions can be characterized by the Craya-Curtet number $C_{t}$. The experiment shows that recirculation occurs when $C_{t}<0.96$. For a given geometry, recirculation as well as adverse pressure gradients can be intensified by reducing the value of $C_{t}$ at the entrance. Five cases of $C_{t}$ were studied, ranging from no to strong recirculation.

The predicted axial mean velocity profiles at two $C_{t}$ numbers are shown and compared with the experimental data in figure 12, where $R$ and $U m$ are the radius of the cylinder and the sectional mean velocity, respectively. Both models are seen to predict very well the upstream evolution of the flow. As for the downstream development, the results of the present model remain in good agreement with experiments while the SKE model underpredicts the centerline velocity decay at all $C_{t}$ numbers.

The variation of the pressure coefficient $C_{p}$ along the duct wall is shown in figure 13. The pressure distribution is governed by the jet entrainment as well as the contraction and expansion of the flow caused by the recirculation bubble. The decrease in the ambient velocity induced by the entrainment gives rise to an adverse pressure gradient, while the contraction of streamlines produces the opposite effect. These two mechanisms interact more intensely with each other as $C_{t}$ decreases and cause the pressure to vary little in the region upstream of the center of the recirculation bubble. However, in the downstream part of the recirculation bubble, the deceleration of the flow sets up an adverse pressure gradient, the slope of which becomes steeper as $C_{t}$ decreases. Therefore, the ability to capture the 
location of the recirculation center will have a direct impact on the prediction of the pressure. Regarding the comparison between predictions and experiments, it is seen that although both models predict the same total pressure rises which are in excellent agreement with the measurements, the present model captures the pressure distribution much better than does the SKE model for all the $C_{t}$ values.

\section{Conclusion}

A new Reynolds stress algebraic equation model has been developed using a truncated constitutive relation. The development of the model is based on the constraints from rapid distortion (rotation) theory and realizability. Therefore, the present model shows the proper lack of a rotation effect on the isotropic turbulence and is fully realizable, i.e., it will not produce unphysical Reynolds stresses for the mean flow field. The model is calibrated by using basic flows (homogeneous shear and channel flows), and then is applied to complex flows. The calculations have been compared with available experimental data. The comparisons show that the present model does provide significant improvement over the standard $k-\varepsilon$ eddy viscosity model and that the present model is as robust and economical as well. This indicates that the present model has good potential to be a practical tool in engineering applications.

\section{Acknowledgements}

The authors are grateful to Dr. A. Shabbir for his calculations of rotating homogeneous shear flows and to Dr. Z. Yang for useful discussion. The work of J.L. Lumley was supported in part by Contract No. AFOSR 89-0226, jointly funded by the U.S. Air Force Office of Scientific Research (Control and Aerospace Programs), and the U.S. Office of Naval Research, and in part by Grant No. F49620-92-J-0038, funded by the U.S. Air Force Office of Scientific Research

\section{References}

Avva, R.K., Smith, C.E. and Singhal, A.K., 1990, "Comparative study of high and low Reynolds number versions of $\mathrm{K}-\varepsilon$ models," AIAA paper 90-0246.

Barchilon, M., and Curtet, R., 1964, "Some details of the structure of an axisymmetric confined jet with backflow," J. Basic Eng., Vol.86, pp.777-787.

Bardina, J., Ferziger, J.H. and Reynolds, W.C., 1983, "Improved turbulence models based on large-eddy simulation of homogeneous incompressible turbulent flows," Rept. No.TF-19, Stanford University, Stanford, Ca.

Chien, K.Y., 1982, "Predictions of channel and boundary-layer flows with a lowReynolds-number turbulence model," AIAA J., Vol.20, pp.33-38.

Driver, D.M. and Seegmiller, H.L., 1985, "Features of a reattaching turbulent shear layer in divergent channel flow," $A I A A$ J., Vol.23, pp.163-171. 
Gatski, T.B. and Speziale, C.G., 1992, "On explicit algebraic stress models for complex turbulent flows," NASA CR 189725 and ICASE Report No.92-58.

Kim, J., Kline, S.J. and Johnston, J.P., 1978, "Investigation of separation and reattachment of a turbulent shear layer: Flow over a backward-facing step," Rept. MD-37, Thermosciences Div., Dept. of Mech. Eng., Stanford University.

Kim, J., 1990, Personal communication.

Lam, C.K.G. and Bremhorst, K., 1981, "A modified form of K- $\varepsilon$ model for predicting wall turbulence," J. Fluids Eng., Vol.103, pp.456-460.

Launder, B.E., Reece, G.J. and Rodi, W., 1975, "Progress in the development of a Reynolds-stress turbulence closure," J. Fluid Mech., Vol.68, pp.537-566.

Launder, B.E. and Sharma, B.I., 1974, "Application of the energy-dissipation model of turbulence to the calculation of a flow near a spinning disk," Letters in Heat and Mass transfer Vol.1, pp.131-138.

Launder, B.E. and Spalding, D.B., 1974, "The numerical computation of turbulent flows," Comput. Methods Appl. Mech. Eng., Vol.3, pp.269-289.

Lumley, J.L., 1978, "Computational modeling of turbulent flows," Adv. Appl. Mech., Vol.18, pp.124-176.

Mansour N.N., Shih, T.-H. and Reynolds, W.C., 1991, "The effect of rotation on initially anisotropic homogeneous flows," Phys. Fluids A, Vol.3, No. 10, pp.2421-2425.

Obi,S., Peric, M. and Scheuerer, G., 1989, "A finite-volume calculation procedure for turbulent flows with second-order closure and co-located variable arrangement," Report. LSTM 276/N/89, Lehrstuhl für Strömungsmechanik, Universität Erlangen-Nürnberg.

Pope, S.B., 1975, "A more general effective-viscosity hypothesis," J. Fluid Mech., Vol.72, pp.331-340.

Reynolds, W.C., 1987, "Fundamentals of turbulence for turbulence modeling and simulation," Lecture Notes for Von Karman Institute.

Rhie, C.M. and Chow, W.L., 1983, "A numerical study of the turbulent flow past an isolated airfoil with trailing edge separation," AIAA J., Vol.21, pp.1525-1532.

Rivlin, R.S., 1955, "Further remarks on the stress deformation relations for isotropic materials," J. Arch. Ratl. Mech. Anal., Vol.4, pp.681-702.

Rodi, W., 1972, "The prediction of free turbulent boundary layers by use of a twoequation model of turbulence," Ph.D. thesis, University of London.

Rodi, W., Majumdar, S. and Schönung, B., 1989, "Finite-volume method for two dimensional incompressible flows with complex boundaries," Comput. Meths. App. Mech. Eng., Vol.75, pp.369-392.

Rubinstein, R. and Barton, J.M., 1990, "Nonlinear Reynolds stress models and the renormalization group," Phys. Fluids A, Vol.2, pp.1472-1476.

Schumann, U., 1977, "Realizability of Reynolds stress turbulence models," Phys. Flu$i d s$, Vol.20, pp.721-725. 
Shih T.-H. and Lumley, J.L., 1992, "Kolmogorov behavior of near-wall turbulence and its application in turbulence modeling," NASA TM 105663, also in Int. J. Comput. Fluid Dynamics, Vol.1, pp.43-56.

Shih, T.-H. and Lumley, J.L., 1993, "Remarks on turbulent constitutive relations," Mathl. Comput. modelling, Vol. 18. No. 2, pp.9-16.

Shuen, J.S., 1992, Private communication.

So, R.M.C. and Lai, Y.G., 1988, "Low-Reynolds-number modelling of flows over a backward-facing step," J. Appl. Math. Phys. (ZAMP), Vol.39, pp.13-27.

Stone, H.L., 1968, "Iterative solution of implicit approximations of multidimensional partial differential equation," SIAM J. Num. Anal., Vol.5, pp.530-558.

Taulbee, D.B., 1992, "An improved algebraic Reynolds stress model and corresponding nonlinear stress model," Phys. Fluids A, Vol.4, pp.2555-2561.

Taulbee, D.B., 1994, "Stress relation for three-dimentional turbulent flows," Phys. Fluids $A$, Vol.6 (3), pp.1399-1401.

Tavoularis, S. and Corrsin, A., 1981, "Experiments in Nearly Homogeneous Trubulent Shear Flow with a Uniform Mean Temerature Gradient," Part I, J. Fluid Mech. Vol.104, pp.311-347.

Thangam, S. and Hur, N., 1991, "A highly-resolved numerical study of turbulent separated flow past a backward-facing step," Int. J. Eng. Sci., Vol.29, pp.607-615.

Yoshizawa, A., 1984, "Statistical analysis of the derivation of the Reynolds stress from its eddy-viscosity representation," Phys. Fluids, Vol.27, pp.1377-1387.

Van Doormal, J.P. and Raithby, G.D., 1984, "Enhancements of the SIMPLE method for predicting incompressible fluid flows," Num. Heat Trans., Vol.7, pp.147-163.

Yang, Z. and Shih, T.-H., 1992, "A new time scale based K- $\varepsilon$ model for near wall turbulence," NASA TM 105768.

Zhu, J., 1991a, "A low diffusive and oscillation-free convection scheme," Comm. App. Num. Methods., Vol.7, pp.225-232.

Zhu, J., 1991b, "FAST-2D: A computer program for numerical simulation of twodimensional incompressible flows with complex boundaries," Rept. No.690, Institute for Hydromechanics, University of Karlsruhe. 

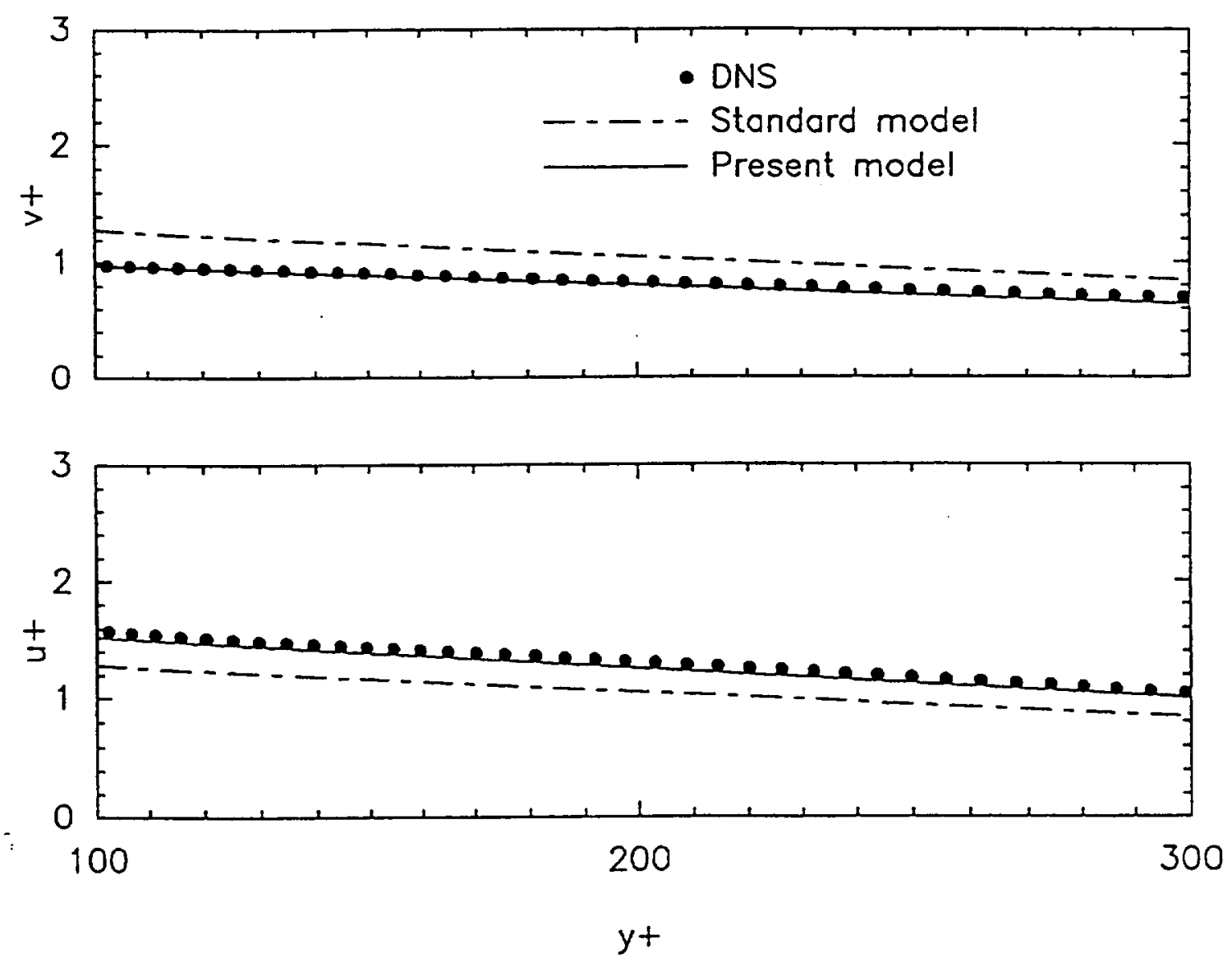

Figure 1 Direct comparison with DNS data of channel flow of Kim 


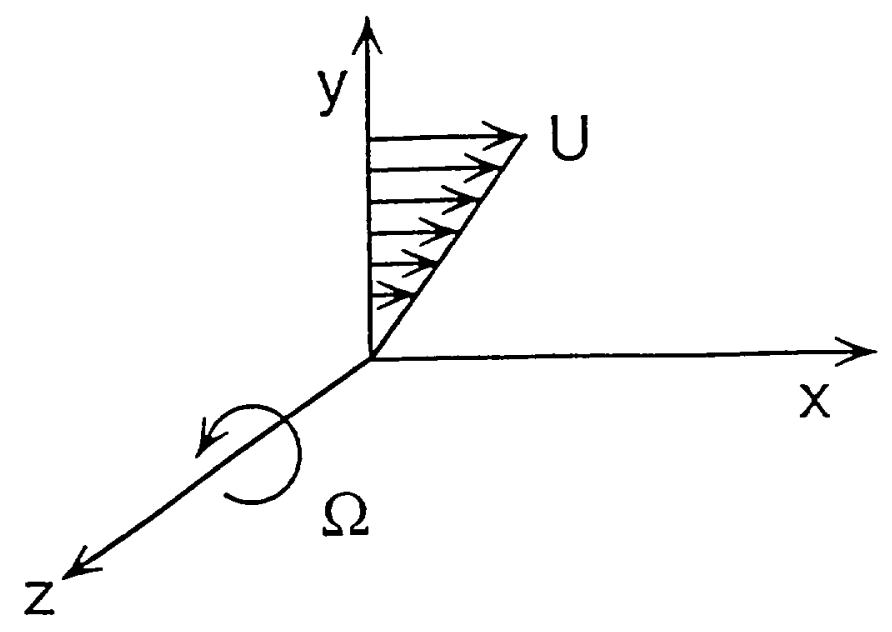

Figure 2 Configuration of rotating homogeneous shear flow

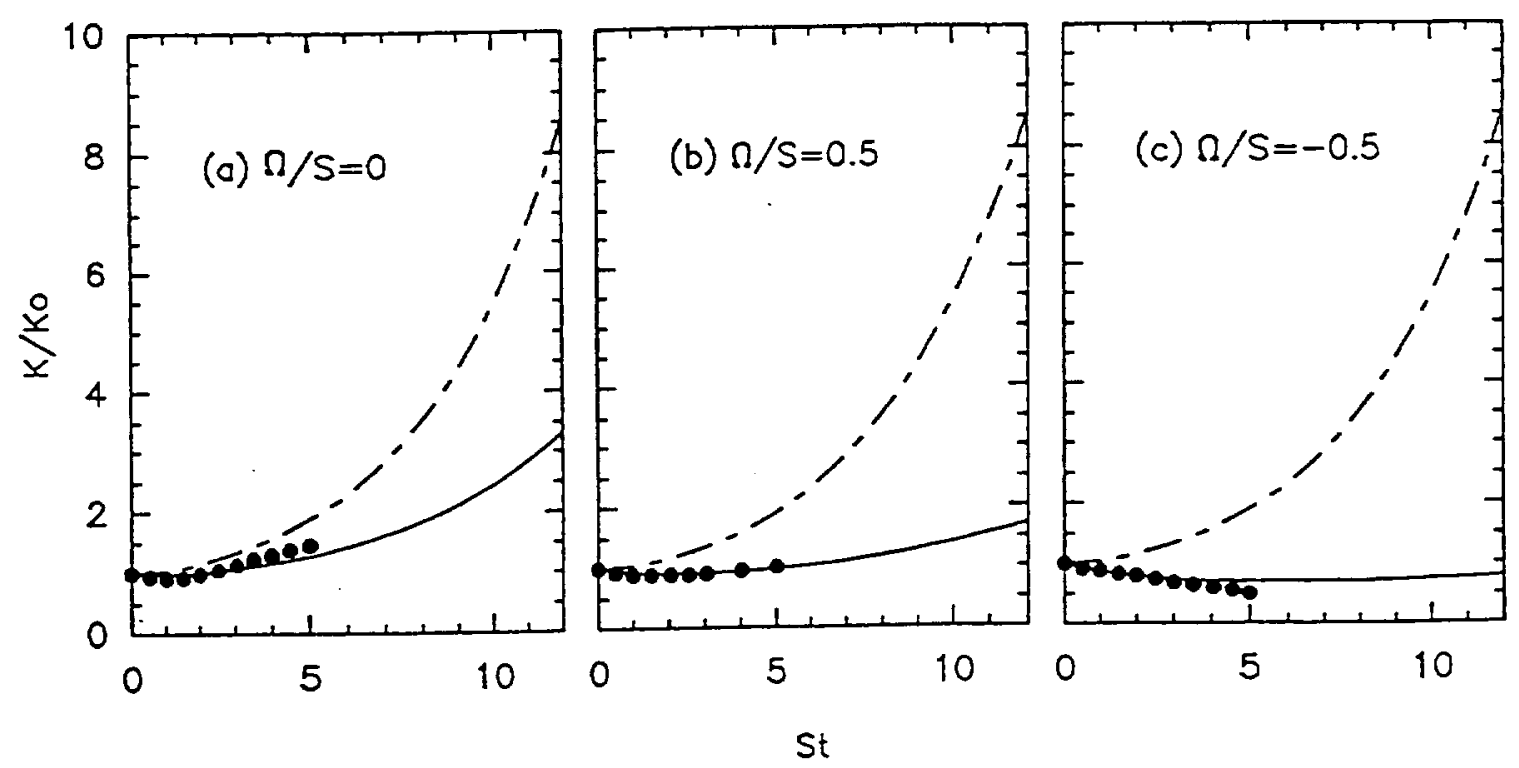

Figure 3 Evolution of turbulent kinetic energy with time.

- : present model; - - : SKE; • : LES 


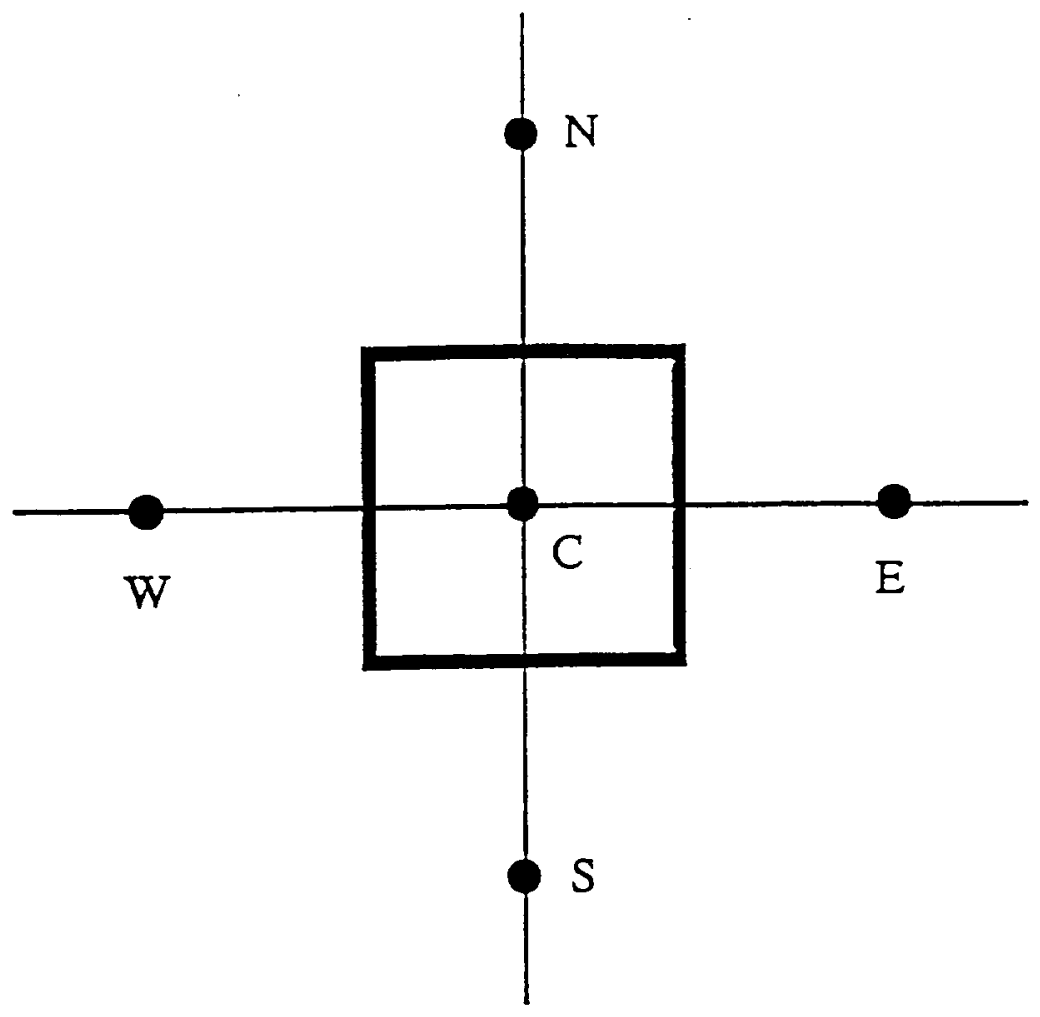

Figure 4 Typical control volume centered at $\mathrm{C}$ and related nodes

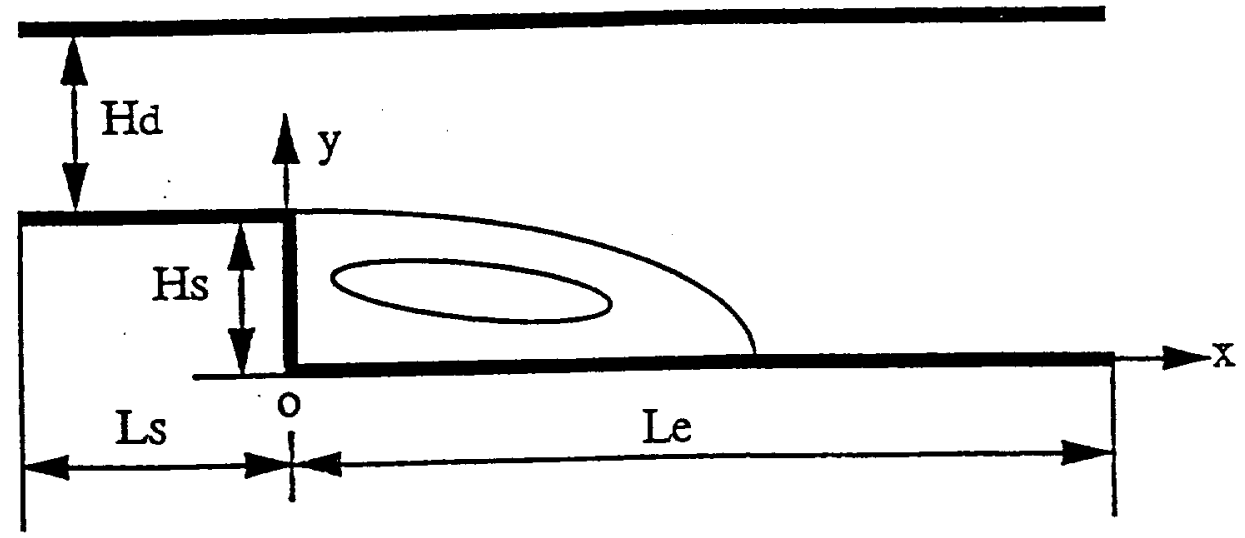

Figure 5 Backward-facing step geometry 

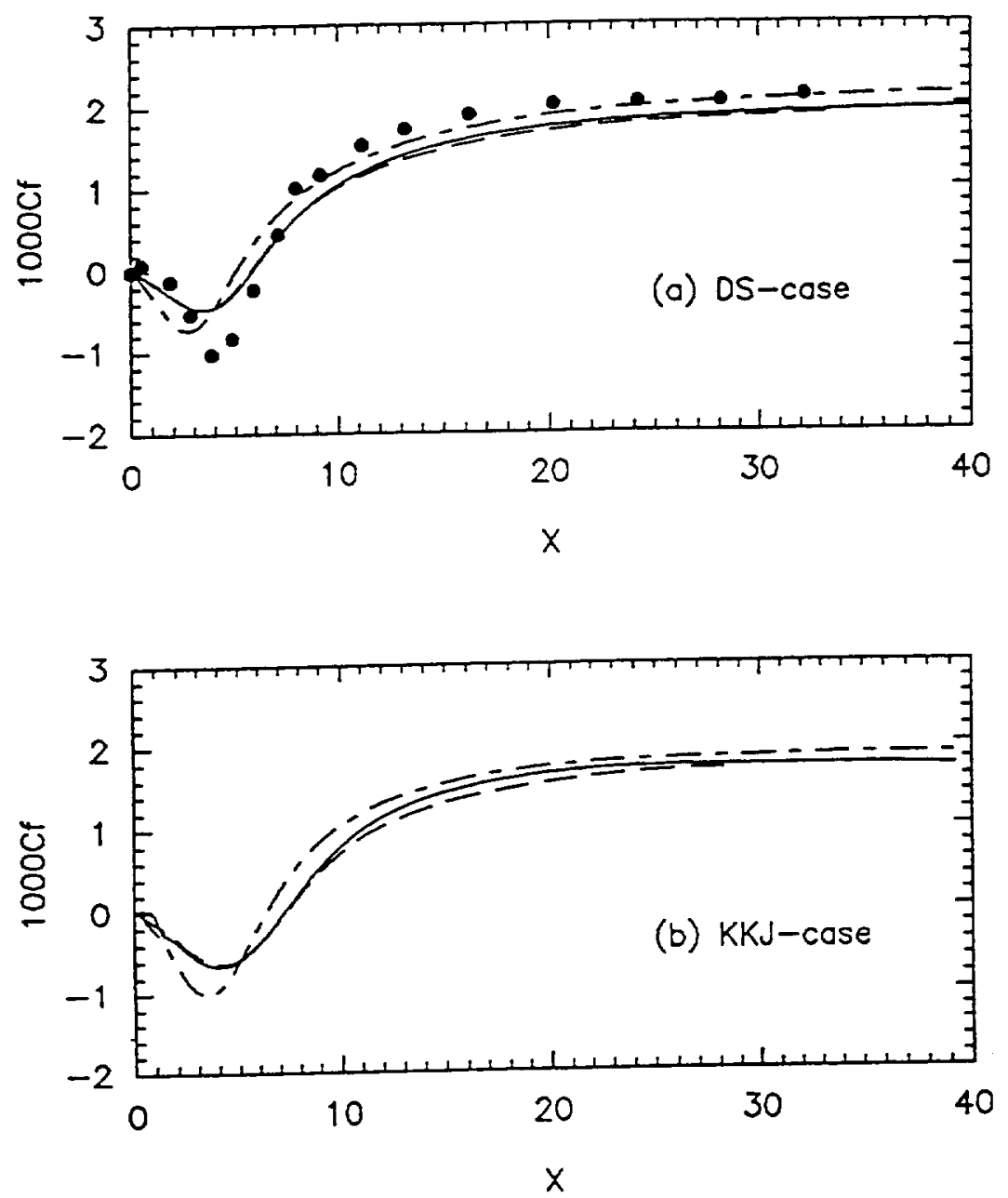

Figure 6 Friction coefficient $C_{f}$ along the bottom wall. - : present model, fine grid; - - : present model, coarse grid; - - : : SKE, fine grid $\bullet:$ : experiment 

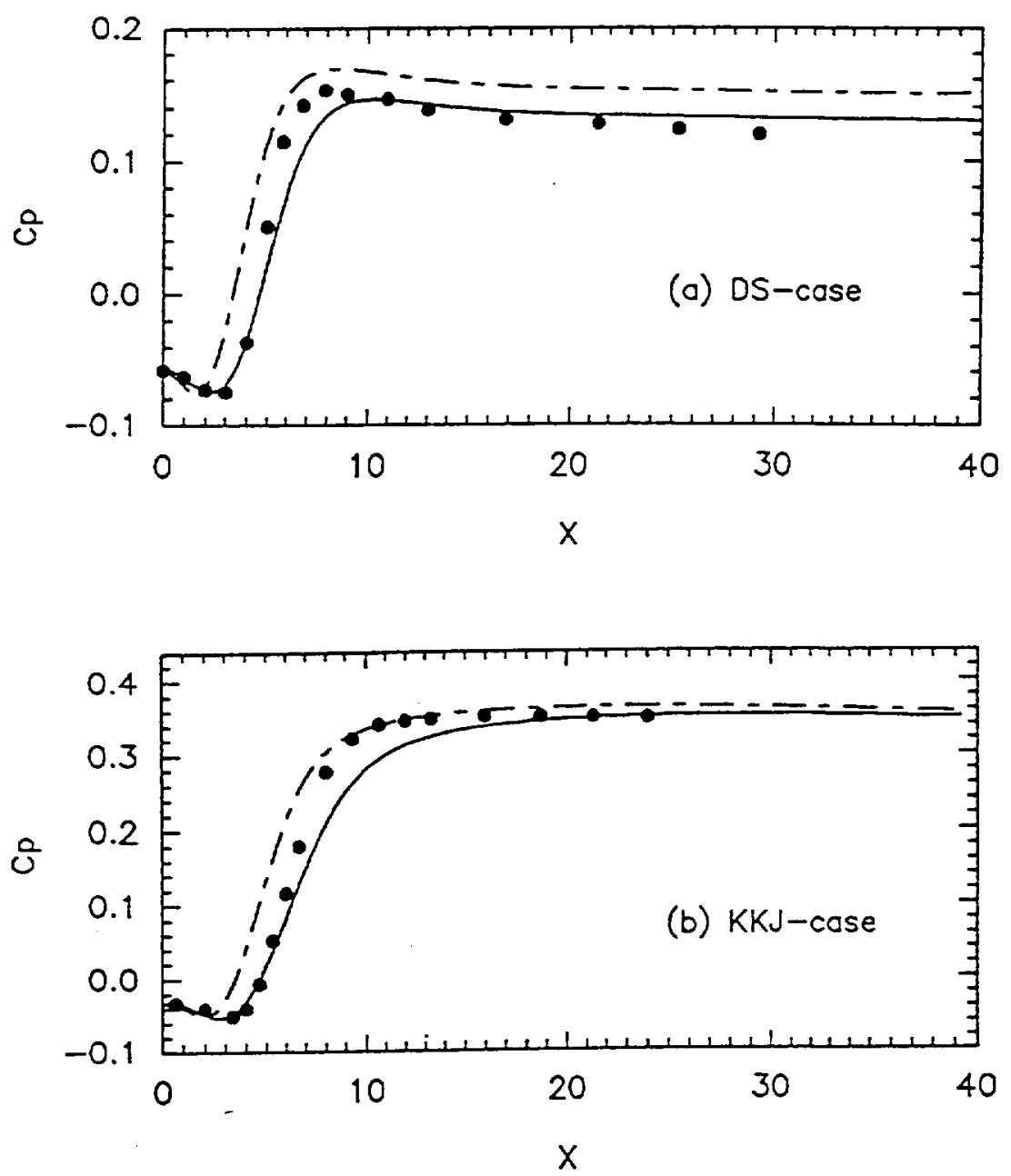

Figure 7 Pressure coefficient along the bottom wall. - : present model; - - - :SKE; $\bullet:$ experiment 

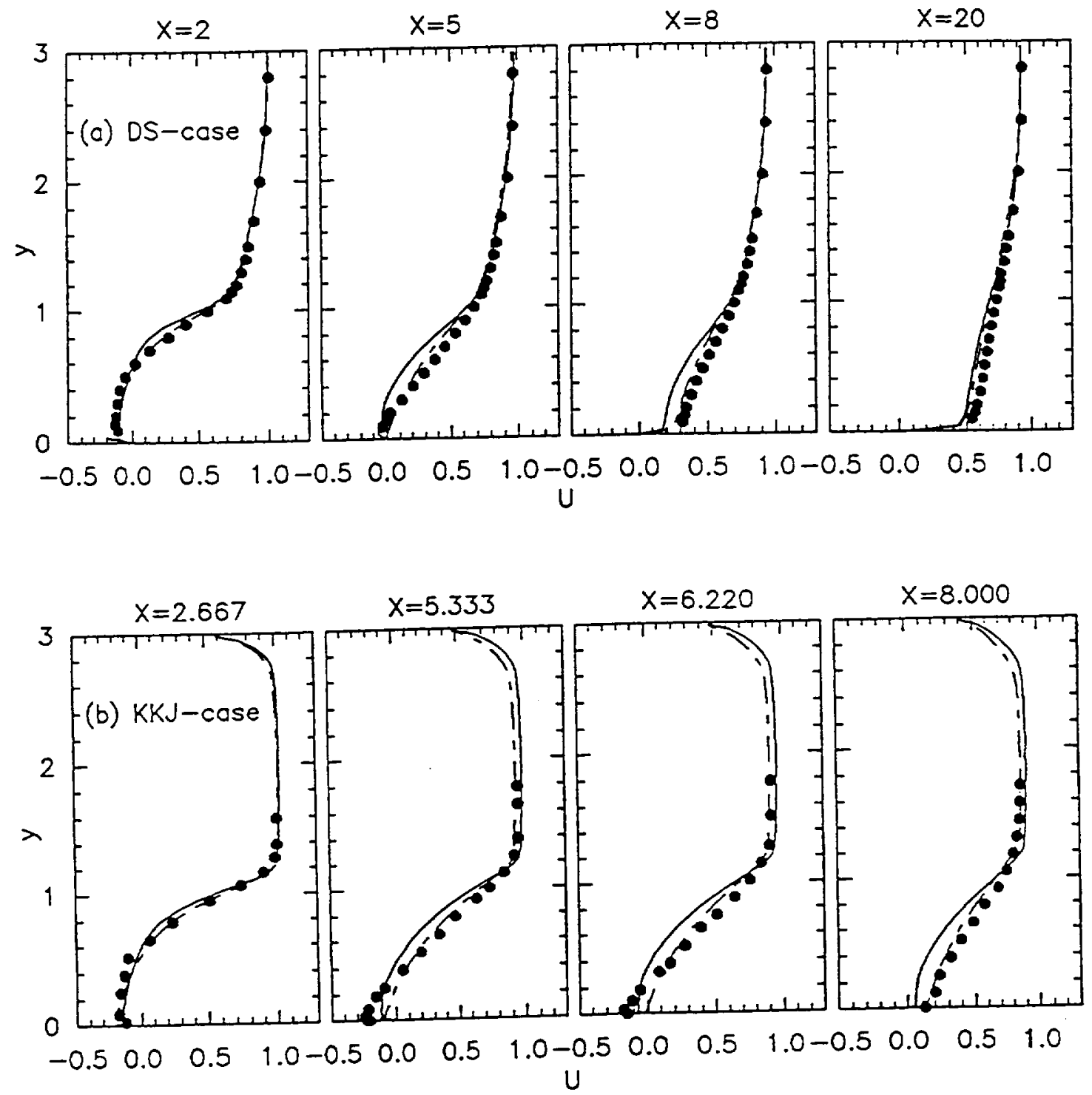

Figure 8 Streamwise mean velocity U-profiles.

- : present model; $---: \mathrm{SKE} ; \bullet:$ experiment 

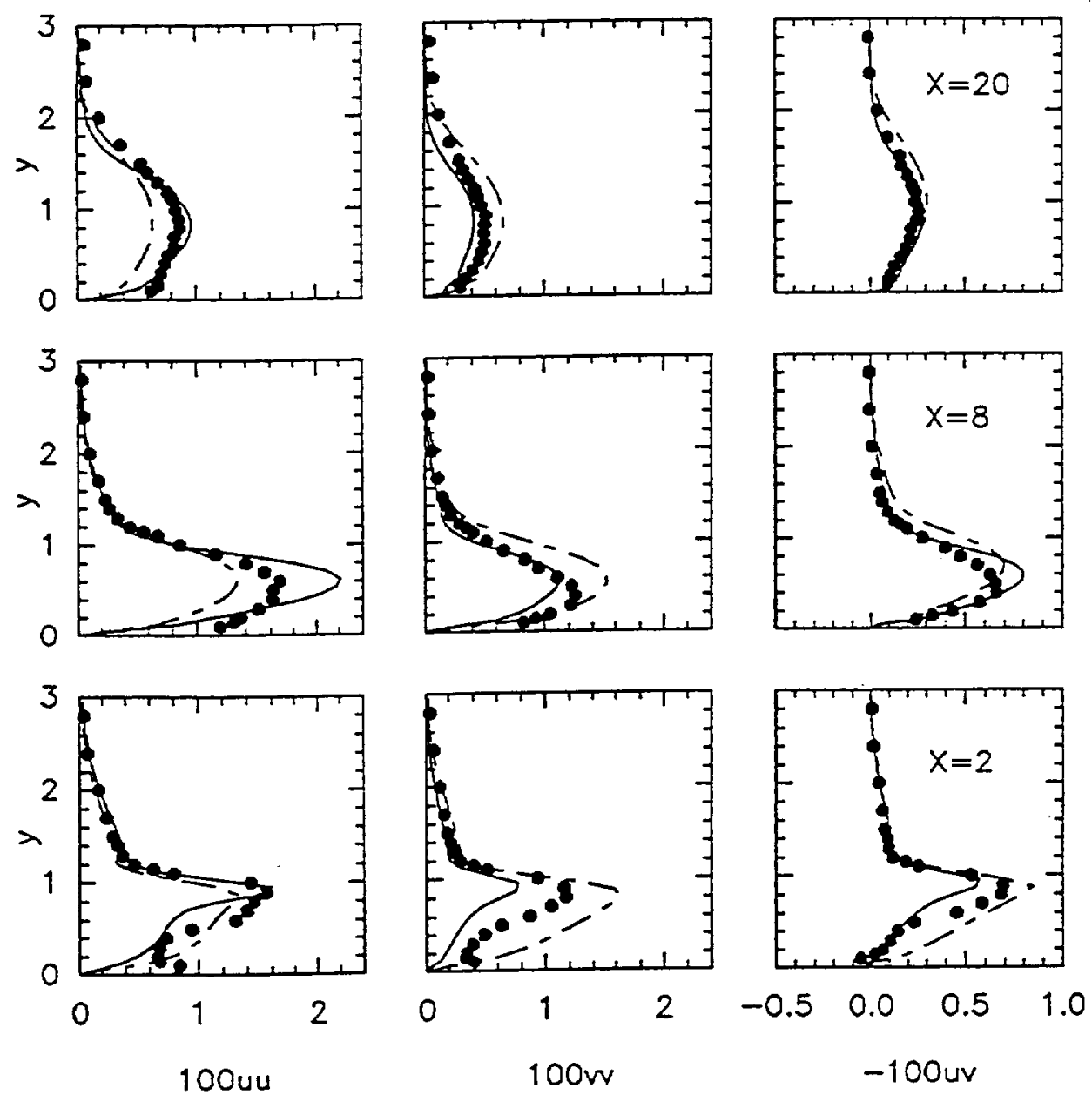

Figure 9 Turbulent stress profiles in DS-case.

— : present model; — _ $:$ SKE; $\bullet$ : experiment 

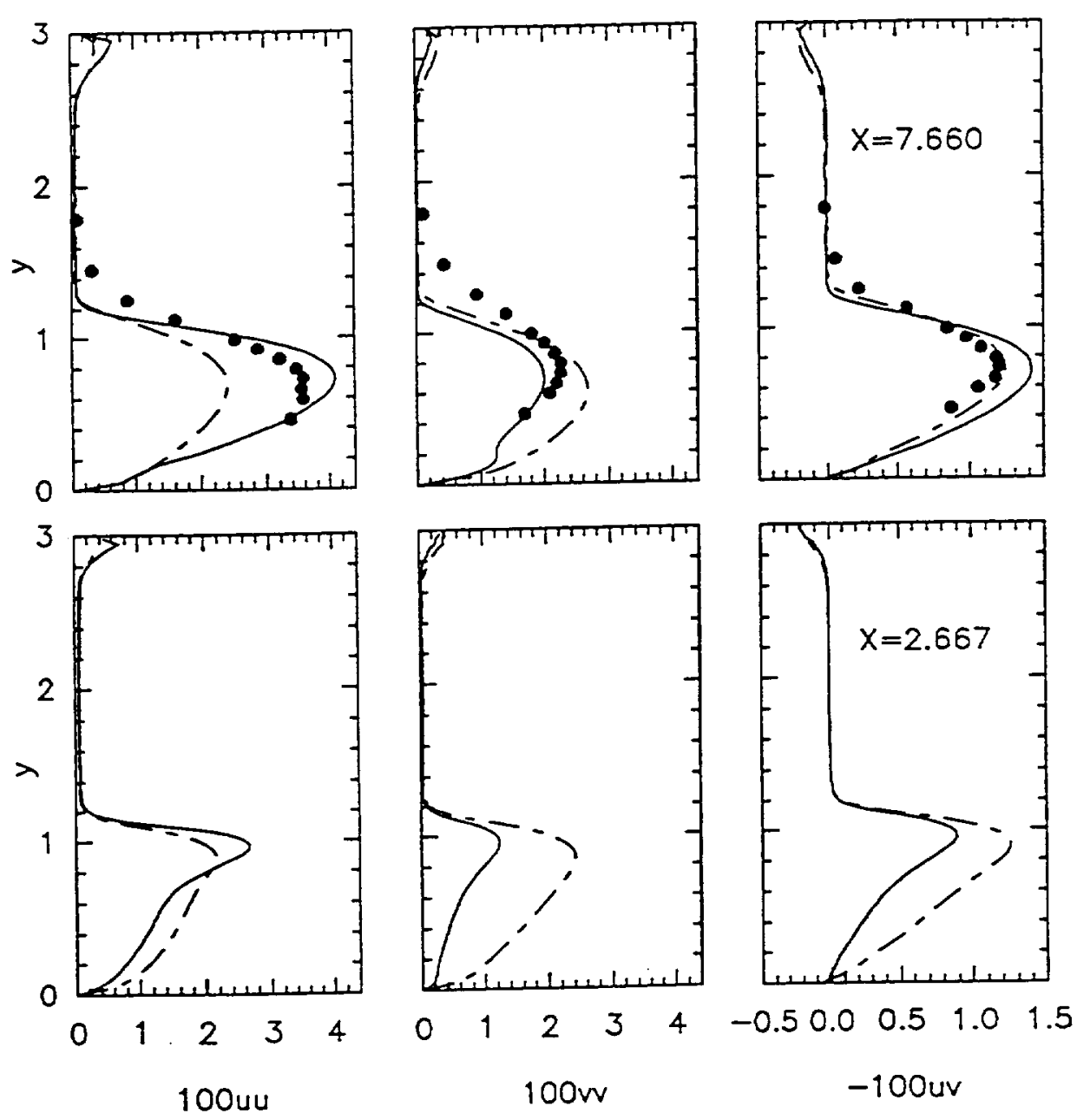

Figure 10 Turbulent stress profiles in KKJ-case.

- : present model; - - - : SKE; $\bullet$ : experiment 


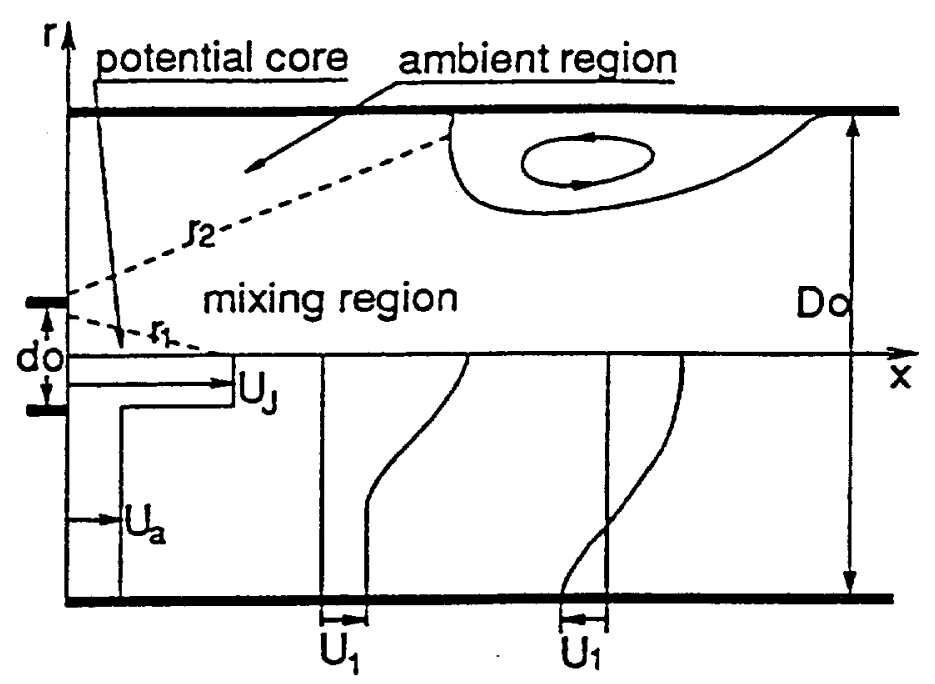

Figure 11 Flow configuration for confined jet 

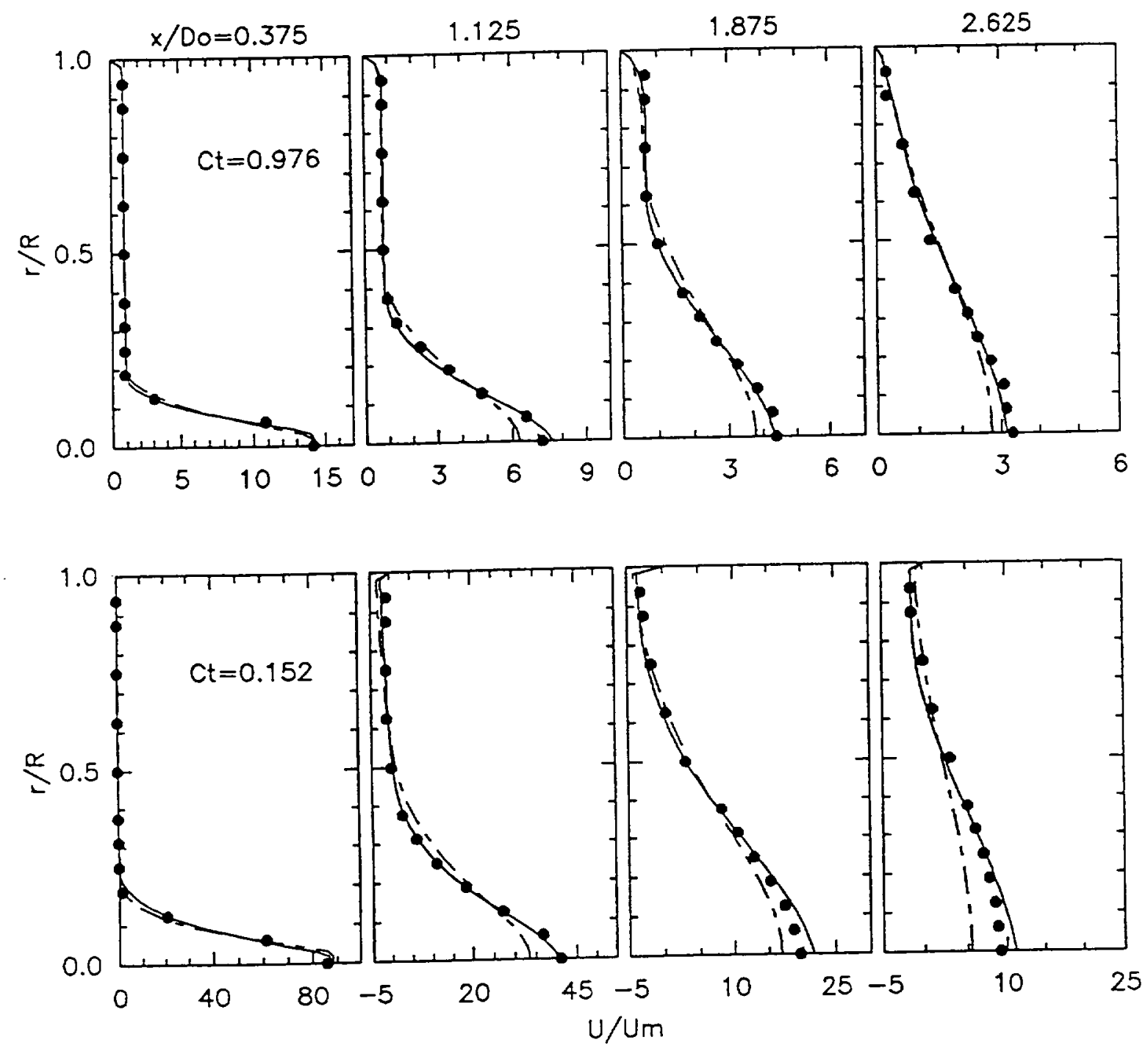

Figure 12 Axial mean velocity profiles.

$\longrightarrow$ : present model; — - - : SKE; $\bullet$ : experiment 


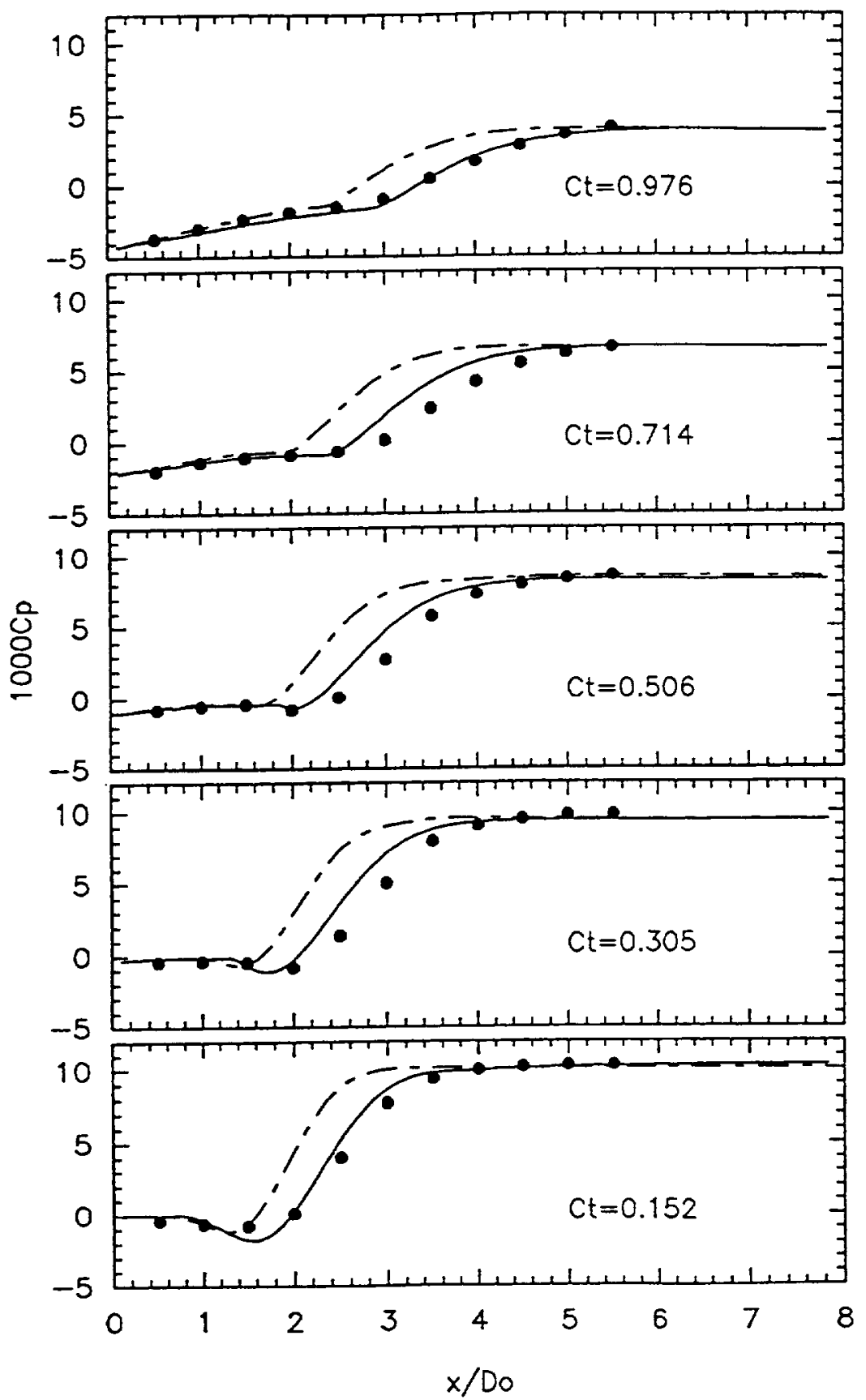

Figure 13 Pressure coefficient along the duct wall. - : present model; $-\cdots$ : SKE; $\bullet$ : experiment 
Public reporting burden for this collection of information is estimated to average 1 hour per response, including the time tor reviewing instructions, searching existing data sources, gathering and maintaining the data needed, and completing and reviewing the collection of information. Send comments regarding this burden estimate or any other aspect of this collection of information, including suggestions for reducing this burden, to Washington Headquarters Services, Directorate for Intormation Operations and Reports, 1215 Jefferson Davis Highway, Suite 1204, Arlington, VA 22202-4302, and to the Office of Management and Budget, Paperwork Reduction Project (0704-0188), Washington, DC 20503.
1. AGENCY USE ONLY (Leave Dlank)
2. REPORT DATE
August 1994
3. REPORT TYPE AND DATES COVERED
Technical Memorandum

4. TITLE AND SUBTITLE

5. FUNDING NUMBERS

A New Reynolds Stress Algebraic Equation Model

6. AUTHOR(S)

WU-505-90-5K

Tsan-Hsing Shih, Jiang Zhu, and John L. Lumley

7. PERFORMING ORGANIZATION NAME(S) AND ADDRESS(ES)

8. PERFORMING ORGANIZATION REPORT NUMBER

National Aeronautics and Space Administration

Lewis Research Center

Cleveland, Ohio 44135-3191

E-8949

9. SPONSORINGMONITORING AGENCY NAME(S) AND ADDRESS(ES)

10. SPONSORING/MONITORING AGENCY REPORT NUMBER

National Aeronautics and Space Administration

Washington, D.C. 20546-0001

NASA TM-106644

ICOMP-94-15

CMOTT-94-8

\section{SUPPLEMENTARY NOTES}

Tsan-Hsing Shih and Jiang Zhu, Institute for Computational Mechanics in Propulsion and Center for Modeling of Turbulence and Transition, NASA Lewis Research Center (work funded under NASA Cooperative Agreement NCC3-233); John L. Lumley, Comell University, Ithaca, New York. ICOMP Program Director, Louis A. Povinelli, organization code 2600, (216) 433-5818.

12a. DISTRIBUTIONVAVAILABILITY STATEMENT 12b. DISTRIBUTION CODE

Unclassified - Unlimited

Subject Category 34

\section{ABSTRACT (Maximum 200 words)}

A general turbulent constitutive relation (Shih and Lumley, 1993, Mathl. Comput. Modelling, Vol. 18, No. 2, pp. 9-16) is directly applied to propose a new Reynolds stress algebraic equation model. In the development of this model, the constraints based on rapid distortion theory and realizability (i.e. the positivity of the normal Reynolds stresses and the Schwarz' inequality between turbulent velocity correlations) are imposed. Model coefficients are calibrated using wellstudied basic flows such as homogenous shear flow and the surface flow in the inertial sublayer. The performance of this model is then tested in complex turbulent flows including the separated flow over a backward-facing step and the flow in a confined jet. The calculation results are encouraging and point to the success of the present model in modeling turbulent flows with complex geometries.

\begin{tabular}{|c|c|}
\hline $\begin{array}{c}\text { 14. SUBJECT TERMS } \\
\text { Turbulence modeling }\end{array}$ & $\begin{array}{c}\text { SEIS PAGE } \\
\text { Unclassified }\end{array}$ \\
\hline $\begin{array}{c}\text { 17. SECURITY CLASSIFICATION } \\
\text { OF REPORT } \\
\text { Unclassified }\end{array}$ & $\begin{array}{c}\text { SECURION } \\
\text { OF THIT CLASSIFICATION }\end{array}$ \\
\hline
\end{tabular}

\begin{tabular}{|c|c|}
\hline & $\begin{array}{l}\text { 15. NUMBER OF PAGES } \\
30\end{array}$ \\
\hline & $\begin{array}{r}\text { 16. PRICE CODE } \\
\text { A03 }\end{array}$ \\
\hline $\begin{array}{l}\text { 19. SECURITY CLASSIFCATION } \\
\text { OF ABSTRACT } \\
\text { Unclassified }\end{array}$ & 20. LIMITATION OF ABSTRACT \\
\hline
\end{tabular}

